\title{
Tangence
}

\section{Marge et marginalité comme modes d'être du discours chez Hubert Aquin}

\section{Claudie Gagné}

Numéro 37, septembre 1992

Autopsie du fait divers

URI : https://id.erudit.org/iderudit/025731ar

DOI : https://doi.org/10.7202/025731ar

Aller au sommaire du numéro

Éditeur(s)

Tangence

ISSN

1189-4563 (imprimé)

1710-0305 (numérique)

Découvrir la revue

Citer cet article

Gagné, C. (1992). Marge et marginalité comme modes d'être du discours chez

Hubert Aquin. Tangence, (37), 107-116. https://doi.org/10.7202/025731ar d'utilisation que vous pouvez consulter en ligne.

https://apropos.erudit.org/fr/usagers/politique-dutilisation/ 


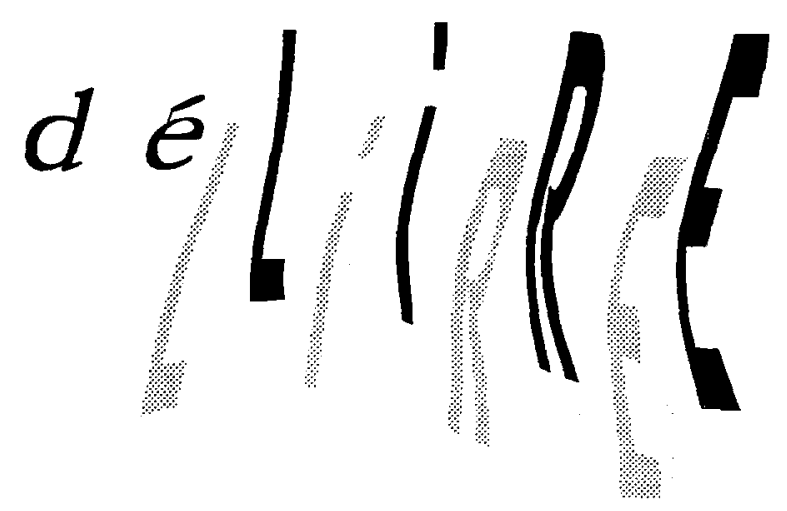

\title{
Marge et marginalité comme modes d'être du discours chez Hubert Aquin
}

\section{Claudie Gagné}

\begin{abstract}
Retrouver les lieux *derrière* les propositions de surface revient donc à reconstituer pour le texte une tout autre atmosphère, d'autres déterminations, une stratégie active mais invisible qui appartient pourtant à la littéralité du discours et non à une extrapolation ou une herméneutique.

Marc Angenot, La parole pamphlétaire, p. 176.
\end{abstract}

Hubert Aquin s'est suicidé le 15 mars 1977: un pauvre quinzième anniversaire cette année. Pourquoi ne pas le souligner au moyen d'un bref retour sur le documentaire de François Ricard et de Jacques Godbout: Deux épisodes dans la vie d'Hubert Aquin? Ce film, en plus de jeter un regard supplémentaire sur les circonstances qui ont conduit l'écrivain à se donner la mort, concerne d'emblée le propos de cette recherche et cible aussi l'attention sur le personnage même de l'auteur (au demeurant fort difficilement séparable de l'œuvre). Au gré du visionnement, la problématique qui y est cernée nous enjoint de constater qu'Hubert Aquin, agissant à titre d'acteur dans une fiction, jouait en partie son propre rôle. Dans ce documentaire, on nous apprend qu'il ira jusqu'à utiliser, plus tard, le nom du personnage (Hubert Deslauriers) pour réserver une chambre d'hôtel où il tentera de mettre à exécution le projet de se suicider. 
De mon point de vue, il s'agit là d'un excellent indicateur du métissage et de l'oscillation entre fiction et réel décelables à la lecture des romans de cet écrivain.

Les textes d'Hubert Aquin sur lesquels j'ai fait porter ma lecture ne font pas partie des textes romanesques de l'écrivain. Il s'agit de deux courts essais. L'essai est une forme littéraire hybride qui assimile, à des degrés infiniment variables, tant la fiction qu'une démarche théorique ou réflexive en regard de la littérature, comme ce sera le cas ici. Ce genre littéraire est également un discours enthymématique en ceci qu'il ne désigne pas nécessairement de manière transparente les concepts qui y sont représentés. Le lieu d'où l'on parle agit à titre de présupposé. À l'énoncé syntagmatique (linéaire) qui nous est donné à lire, se superposent tous les paradigmes (les références à d'autres lieux idéologiques, l'intertexte). Ce que je me propose d'observer chez Hubert Aquin, ce sont quelques-uns de ces présupposés qui s'inscrivent précisément dans la marge ou dans les blancs du texte. Les essais que j'ai sélectionnés, "Profession: écrivain ${ }^{1}$ et "Le texte ou le silence marginal? " ${ }^{2}$, traitent de la pratique même de l'écriture, voire de l'-engagement. vis-à-vis de l'écriture, comme le révèlent fort justement les titres.

\section{Aspects du paratexte: " Profession: écrivain *}

Dans le premier texte, le titre "Profession: écrivain" donne déjà la couleur du discours qui sera plus largement développé dans l'article. Il convient, d'ailleurs, d'analyser ce titre. En effet, pouvons-nous concevoir que le métier d'écrivain puisse faire accéder à un statut professionnel? Il faut en douter, surtout de nos jours. À ma connaissance, les seuls lieux où l'artiste-écrivain se voit attribuer un statut professionnel sont les instances gouvernementales qui décernent des bourses de soutien à la pratique artistique: le Conseil des Arts du Canada, le ministère des Affaires culturelles du Québec. L'inscription du substantif "écrivain" à la case "profession " d'un passeport canadien me semble presque constituer une antinomie, voire une percée dans le mythe du

1 Dans l'anthologie de Laurent Mailhot, Essais québécois 1837-1983, p. $438-443$.

2 Hubert Aquin, Blocs erratiques, Montréal, Quinze, coll. ×10/10 •, 1982, p. 269-272. 
héros tragique. Peut-on, en effet, imaginer un formulaire qui inspirerait à un citoyen une telle marque d'individuation? Il suffit de penser à l'exiguîté de l'espace pour décliner son identité. Lorsque, dans un formulaire gouvernemental, on s'enquiert de votre profession, vous répondez de bon ou de mauvais gré, selon que vous appréhendez une réaction positive ou négative à votre réponse. Cette question, plutôt rhétorique, n'interroge pas véritablement; elle vous révèle plutôt la précarité de la place que vous occupez dans la société. Mais le narrateur de *Profession: écrivain ", qui y a répondu sur un mode presque fatidique, aurait tout aussi bien pu se dire rédacteur ou journaliste, fonctions plus acceptables socialement... en tout cas moins connotées idéologiquement que celle qu'il a préféré déclarer. Ainsi, le seul titre tiendrait du pamphlet. On sait qu'à cette époque (1960-1968), Aquin était engagé dans le R.I.N. et dans l'édition, à la rédaction de la revue Liberté. Et malgré tout, le ministère des Affaires extérieures du Canada aurait autorisé l'émission d'un passeport portant l'inscription "écrivain". Comme si ce type d'interrogation ("Nom? Profession? "), dans la mesure où vous présentez force documents en règle, ne faisait que retourner à votre conscience une sous-question qui pourrait se formuler ainsi: quelle image professionnelle désirez-vous présenter de vous-même à l'étranger? Or, n'est-ce pas se poser en "étranger " que de se déclarer écrivain? Voici un extrait qui permet cette interprétation:

Depuis que j'ai fait inscrire cela dans mon passeport, je n'ai pas cessé de commettre des sacrilèges contre cette investiture consulaire, à tel point que j'en suis arrivé à me réjouir de tricher avec ma vocation et même à me transformer en non-écrivain absolu. 3

\section{Marginalité au deuxième degré}

L'énonciateur se donne pour marginal vis-à-vis de la profession d'écrivain qu'il affiche. Mais il s'agit en fait d'un double rapport de marginalité, plus exactement d'une marginalité au deuxième degré. Comme je l'ai mentionné plus haut, ce n'est pas faire preuve de conformisme que de se dire écrivain dans les cases d'un formulaire gouvernemental. L'écriture littéraire n'est pas une profession comme la rédaction peut en être une,

-Profession: écrivain *, p. 438. 
facilement admise, voire essentielle selon le sens commun. Elle impliquerait plutôt une liberté absolue, totale: toute norme sociale ne peut-elle pas être transgressée dans l'écriture? Celle-ci est rigoureusement individuelle et asociale, amorale même, contrairement à la rédaction journalistique, par exemple, qui est régie par plusieurs codes: code de déontologie, code professionnel (on travaille pour un patron qui paie un salaire), etc. La charge idéologique d'un discours de journaliste se trouve toujours, en tout cas dans une large mesure, entérinée par la direction d'un journal. Mais l'écrivain, quoique pourvu d'un pouvoir social minime, est doté d'un pouvoir linguistique sans bornes. Faut-il reconnaître là un rapport de cause à effet? Il peut décider des paroles et des actes de ses personnages. Son pouvoir est incontestablement démiurgique. D'où la connotation pamphlétaire de cette seule déclaration "Profession: écrivain " par un Québécois francophone minoritaire et folklorisé dans le contexte canadien.

Dans "Profession: écrivain*, le sujet de l'écriture met en procès cette profession d'écrivain inscrite dans son passeport. Il rejette cette réalité et tente de "tricher avec sa vocation ", comme s'il voulait se tenir à l'écart de l'engagement que le seul fait de prendre la plume suppose. Pendant un moment, il est fier des activités qui le constituent écrivain; l'instant d'après, alors qu'il réfléchit à son geste, il s'insurge contre cette irrémédiable inscription comme si elle était en fait le signe d'une tare. Le passage qui suit conforte cette hypothèse, car on assiste à la tentative délibérée du narrateur de se marginaliser face à l'État par une affirmation publique et politique de son statut d'écrivain:

Mais, le temps de changer de profession ailleurs que sur mon passeport, j'ai dû me rendre à l'évidence que, pour mes interlocuteurs, mes activitês antérieures me constituent définitivement en homme de lettres. Quelques commandes de textes, une incorporation irréversible, en quelque sorte, à la Société des Auteurs: il a fallu aussi peu que cela pour me rappeler que je suis désormais - et en dépit de mes dénégations et de mes dispersions - engrené sans huile dans une mécanique qui me remet à ma place. ${ }^{4}$

Dans cet extrait, on le voit tour à tour abandonner son travail littéraire, se désister de son rôle et de sa position sociale dès qu'il 
se croit reconnu par les autres en tant qu'écrivain. Ce fragment constitue un topos très important du texte, car à lui seul il condense les dualités, dichotomies ou ambivalences de la posture marginale du narrateur, situation rarement confortable, le plus souvent insoutenable.

\section{Résignation à l'écriture ?}

Pour le narrateur, il s'agirait presque d'un retour à la fois résigné et résolu à l'écriture. Posture paradoxale, à vrai dire, que d'assumer ce mal dont il n'espère même plus se guérir, condamné d'avance par l'institution littéraire comme le Juif par la dictature nazie. Le narrateur se sent Juif, et convoque ici l'une des plus saisissantes métaphores de la répression sociale: * je me sens comme un Juif en cela que le regard des autres me rend Juif " 5 . S'appuyant sur cette image du rejet, le sujet de l'écriture souligne sa mise à l'écart de la société et rend compte de la marginalisation qu'il subit. Cette référence au nazisme surdétermine la correspondance que le narrateur veut établir entre, d'une part, la situation de l'écrivain québécois dans le contexte canadien, et, d'autre part, la situation de l'écrivain face à la société en général.

Peut-on encore parler de résignation, de résolution à l'écriture? Non, pas tout à fait, puisque, dans la suite du texte, le narrateur lui résiste encore, accablant d'invectives la langue française:

Mais si la fortune ou ma paresse ne m'éjectent pas hors de mon siège social d'écrivain, j'ai l'intention de faire payer cher à sa majesté ma langue à moitié morte, mon incarcération syntaxique et l'asphyxie qui me menace; oui, je projette de me venger sur les mots déliés, de cette belle carrière qui s'ouvre devant moi à la manière d'une mine qui se referme sur celui qui l'approfondit. Je suis la proie de pulsions destructrices contre cette méchante langue française, majestueuse bien sûr mais seconde! Écrire me tue. 6

Ici, on passe de la situation sociale de l'écrivain à la question de son rapport avec la langue même. Les indices de marginalité cohabitent nombreux dans ce passage. L'énonciateur annonce la vengeance qu'il entend exercer sur celle qui l'a logé à l'enseigne 
institutionnelle de la littérature, à savoir cette toute puissante (quoique dominée) langue française. En effet, il signifie son intention d'utiliser tous les moyens à sa disposition pour mener jusqu'au bout sa rébellion. Le scripteur manifeste son refus de toute incarcération dans la syntaxe, comme il s'oppose à toute résignation, à l'asphyxie. Le camp de concentration, c'est la langue.

Le fait d'être écrivain et de s'en réclamer ouvertement n'arrime pas moins le sujet écrivant au milieu carcéral du langage. Cette métaphore pénitencière n'est pas innocente, puisqu'elle est filée (voire prise en filature) tout au long de l'article. L'image rend également compte de la pression du regard que les autres portent sur quelqu'un qui se dit écrivain. Elle met en relief le prix à payer pour écrire, prix beaucoup trop élevé pour le scripteur. Résumons: l'écrivain est un être marginal par définition. Lorsqu'il veut échapper à la doxa inhérente à la langue, il se marginalise une deuxième fois. Et s'il poussait à fond sa rébellion, son écriture se commettrait dans une éthique de la transgression des codes langagiers. Donc, de la transgression de toute idéologie.

\section{Une énonciation marginale : l'incohérence}

Selon Aquin, s'exprimer hors de la doxa est criminel: aussi utilisera-t-il la formule de "crime verbal "7. C'est ainsi qu'il commence à plaider en faveur d'une pratique de l'incohérence comme principal facteur dynamique de son projet d'écriture. Le scripteur s'inscrit en faux contre la mission première de l'écrivain, celle de faire signifier les mots, et revendique le droit de verser dans l'illogisme. Pour signifier son intention d'* incohérer * (néologisme utilisé textuellement dans *Profession: écrivain *), il fait intervenir l'intertexte. Pas n'importe lequel, un texte canonique, Hamlet: "words words words 8 . Sombrer dans l'incohérence sur le mode hamlétien contribue à élargir le "spectre " de l'interprétation. Dans sa volonté de remuer les mots sans but, l'écrivain énonce, lorsqu'il convoque Hamlet par exemple, qu'il faut se méfier de la fausse transparence et redouter l'opacité d'une pensée intimement liée à l'écriture. Cette écriture, issue des marges d'un autre texte, cherche à révéler quelque chose, à l'état de 
présence dans la négation, suis-je tentée de dire: quelque chose que le narrateur refuse de donner de prime abord, mais dont il cherche à nous donner le contexte, que l'on sera sans doute en mesure de percevoir au terme de la lecture. Tout se passe comme si l'inconscient du texte voulait révéler l'imposture. Quelle imposture? Celle qui associe l'écrivain à la classe dominante? Celle qui sacralise tel objet culturel davantage pour sa valeur d'échange que pour sa valeur d'usage? Qu'en est-il exactement? Pour le narrateur, écrire dans le respect absolu du code (syntaxe correcte, fidèle véhicule d'une pensée linéaire) signifie qu'on ne peut échapper au schéma de la domination, puisque de cette façon *le manieur de mots" se voit contraint à perpétuer ce schéma. Voilà bien la situation piégée de l'écrivain. Sa pratique demeure à jamais marquée au coin de la domination, et se voit obligée de renoncer à la gratuité esthétique et formelle.

L'important est-il que je sois doué pour les arts? Non, mais de savoir que je suis doué pour les arts du fait même que je suis dominé, que tout mon peuple est dominé et que son dominateur l'aime bien tzigane, chantant, artiste jusqu'au bout des doigts, porté tout naturellement vers les activités sociales les plus déficitaires. Au fond, je refuse d'écrire des œuvres d'art, après des années de conditionnement dans ce sens, parce que je refuse la signification que prend l'art dans un monde équivoque. Artiste je jouerais le rôle qu'on m'a attribué: celui du dominé qui a du talent. 9

Comment écrire lorsqu'on refuse de satisfaire les attentes d'un Surmoi social qui décrète de façon implicite que *le talent du dominé provient de l'envie de faire la révolution n'importe comment en art, faute de pouvoir la faire en histoire "10? Pourquoi l'écriture, si celle-ci n'est que le symptôme d'une pulsion révolutionnaire refoulée dans le langage?

En fait, le discours du narrateur consiste à vouloir d'abord trangresser les normes imposées, et à faire exploser ce qu'elles tentent d'expurger du sujet écrivant. Le scripteur cherche à conjurer sa condition de dominé et à exorciser l'emprise des normes passéistes sur son écriture. D'où cette incohérence dans le texte du scripteur. D'une part, il importe de faire crisser la plume sur le papier sans faire démonstration du moindre talent, 
d'autre part, de puiser plus ou moins consciemment au registre d'autres auteurs et, pourquoi pas, de produire des textes illisibles ${ }^{11}$. Sont ainsi déjouées toute prise sémiologique et, par là même, toute tentative de folklorisation. Il s'agit également de décevoir les lecteurs qui seraient tentés d'assimiler la démarche littéraire à une cause sociale ou nationale qui l'asservirait. L'incohérence ne devient-elle pas esthétique, quand on sait qu'Hubert Aquin, dès 1969, sera l'un des premiers professeurs du baroque dans la littérature moderne au Québec? Éloge de l'incohérence, donc, pour jouer au fou (comme Hamlet) face à l'Ordre colonial, pour retourner cet ordre caduc dans tous les sens, le mettre en abyme et le débarrasser de ses vieilles formes. Quitte à ce que *le révolutionnaire choisisse d'être taxé de folie comme le sweet prince du royaume pourri ${ }^{12}$. Le narrateur se situe dans la marge parce que la littérature est nécessairement placée sous le signe de la résignation. N'oublions pas que "Profession: écrivain", rédigé en 1964 pour Parti pris, ne précède que de six mois le séjour d'Hubert Aquin à la Prison de Montréal pour participation à des activités révolutionnaires.

La révolution qui opère mystérieusement en chacun de nous débalance l'ancienne langue française, fait éclater ses structures héritées qui, par la rigueur même de ceux qui les respectaient, exerçaient une hégémonie unilatérale sur les esprits. ${ }^{13}$

\section{L'écriture paradoxale comme écriture de la marge}

Pour Aquin, une œuvre conforme à la doxa n'est pas valable. Elle devrait au contraire tendre vers le paradoxe. En effet, ce serait davantage à son imprévisibilité absolue et à son irrésolution que l'on reconnaîtrait la validité d'une ouvre littéraire. Toute œuvre de qualité ne devrait pas non plus chercher à effacer les traces de souillure de "l'intolérable quotidienneté de notre vie collective " ${ }^{14}$ qui maculent cette langue brisée, marginalisée. L'écriture tout entière se génère dans la marge, se donne lieu dans la marge; l'en retirer signifierait, pour le texte littéraire, un renoncement à son existence. Selon le narrateur, "le néant

11 Expression empruntêe à Roland Barthes qui désigne un texte apte à déployer un pluriel de sens.

12 Ibid., p. 441.

13 Ibid., p. 443.

14 Ibid. 
distingue, tout comme [c'est] la marge [qui] invente le texte 15 . Cette marge matériellement typographiée ou simplement évoquée devient pour le narrateur "la seule structure décelable de tout texte imprimé.16. À la fois embrayeur et destin inavouable de l'écriture, la marge, admise comme élément constitutif du texte, peut constituer un élément moteur dans la transformation d'un discours. Pour le scripteur, peu importe que la marge soit condamnée ou sublimée, ce n'est jamais qu'elle qui module le texte: "[L'écritl ne [tiendrait] donc qu'à sa propre marge "17. Car le texte, contrairement à ce que l'on croit, n'est pas maître sur la page qu'il occupe; et elle-même ne serait jamais tout à fait vierge. Marge et texte coexistent sur la surface de la page, pacifiques ou vindicatifs. En effet, bien que la marge puisse paraitre silencieuse, elle représente une instance oppressante pour l'écriture, justement parce qu'elle rend sa liberté conditionnelle. Et c'est le texte, finalement, qui surgirait en tant que résultante de ce schisme primordial. Combat auquel nous assistons, simples lecteurs, comme si, hors de portée du scripteur, il avait encore lieu sous nos yeux.

\section{Conclusion}

L'écriture d'Hubert Aquin, que plusieurs critiques ont qualifiée de postmoderne, pourrait aussi être étudiée dans ses procédés littéraires: l'analyse de l'autoréflexivité, de l'autoréférentialité et de l'autoreprésentation (la mise en abyme) seraient pertinentes dans la mesure où elles servent à évaluer l'autonomie d'un texte. Si ces prémisses sont valables, il n'y aurait qu'un pas à faire pour énoncer que le texte serait lui-même marginal face à son propre scripteur parce qu'il ne témoigne pas du référent, mais de sa propre genèse (l'intertexte hamlétien).

Peut-être serait-il pertinent de mentionner, en conclusion, celui que les théoriciens d'aujourd'hui ont porté aux nues comme figure emblématique de la rupture d'avec le Romantisme, Flaubert, qui, à l'encontre des principes esthétiques de ses contemporains (écrire pour dire quelque chose, ou écrire parce qu'on a quelque chose à dire), avait l'ambition de parvenir à écrire un jour un livre sur rien. Le rien: sujet provocant s'il en est,

15 "Le texte ou le silence marginal ", p. 270.

16 Ibid.

17 Ibid., p. 271. 
116

en regard des motivations littéraires plus "nobles * qui visaient à donner aux lecteurs une peinture du réel. Flaubert est reconnu aujourd'hui comme le père de la modernité, mais au XIX' siècle ne s'était-il pas attiré l'opprobre de la bonne société? 